International Journal of Pure and Applied Physics

ISSN 0973-1776 Volume 5, Number 2 (2009), pp. 121-132

(C) Research India Publications

https://dx.doi.org/10.37622/IJPAP/5.2.2009.121-132

\title{
Vortex Formation in Axion Condensate and Dynamics of Dark Matter
}

\author{
*R. Sharma, G.K. Upadhyaya and M. Thakrey \\ School of Studies in Physics \\ Vikram University Ujjain(M.P.)456010 INDIA.
}

\begin{abstract}
We examine Dark Matter dynamics by analyzing its fluid properties of vortex formation. Our results show that kinetic energy of a spiral shape galaxy like Milkyway becomes constant at larger distances. It is the same what velocity dispersion curves shows initially in the work of F.Zwicky[1].Our results also fit well with the results of Jakub Mielczarek et al[24].Their exploration shows the vortex formation in the axion condensate and its effect on spiral shape galaxies. In present paper we compare our result with vorticity of Axion condensate.
\end{abstract}

Key Words: Dark Matter.

PACS No.: 95.35. +d

\section{Introduction}

There is a consensus in the astronomer community that most of the mass in our galaxy and of most galaxies, is in the form of dark matter. Dark Matter is the stuff between visible objects. Presence of Dark Matter is inferred by its influence on visible bodies [18].

Over 74 year ago Fritz Zwicky [1] showed that the speed of galaxies in large clusters, such as Coma cluster, is much too great to keep them gravitationally bond unless they weigh over one hundred times more than one would estimate based on the number of stars in cluster. The speed of stars tells that about $80 \%$ of mass of galaxy is invisible. In 1970s further evidence for Dark Matter was found from gravitational studies of matter in the outer parts of ordinary nearby galaxies [2, 3, 4, 5].It invokes upon the idea that there must be a mysterious halo of dark matter that engulfs each galaxy. If there were no dark matter in a galaxy, then the force of gravity would be less and the stars in the outer reaches of a galaxy would fly off into intergalactic 
space. Hence dark matter plays a crucial role in holding galactic objects together. Although dark matter is known to dominate mass budgets of bound systems such as galaxies or galaxy clusters, its nature continues to remain mysterious. On cosmological scales ( $>1 \mathrm{Mpc})$, the "Cold Dark Matter" (CDM) model in which the dark matter is non baryonic, non relativistic and collisionless, had considerable success in matching the observations. Cold Dark Matter is considered because of three basic reasons:

(1) Numerical simulation of structure formation with CDM agrees with most observations of the structure.

(2) CDM well explain the abundance of WIMPs.

(3) CDM contains specific appealing candidates such as neutralino, axions etc. for Dark Matter particles in models of fundamental physics.

In concordance model the nature of Dark Matters well explained at larger scale (Mpc order). But on smaller scale the behavior of Dark Matter is complex and difficult to compute. The principal purported problems found on smaller scales are as follows:

(A) Substructure: Small halos and galaxies orbiting within large units may not be as common as expected on the basis of numerical simulation of CDM $[6,7,8]$.

(B) The density profile of Dark Matter halos should exhibit a cuspy core in which the density rises sharply as the distance from the center decreases, in contrast to central regions of many observed self-gravitating system $[9,10,11,12,13$, 14].

The possible discrepancies between theory and observation have motivated new proposals for the nature of Dark Matter. Each proposed variation from standard cold, collisionless Dark Matter (CCDM) has two properties:

- it can solve some or all problems described earlier, and

- it leads to additional predictions that would distinguish it from all the other alterations.

There are lots of approaches present in the literature to understand viscosity, sticky nature of Dark Matter we focus our work on understanding the fluid type nature of the dark matter $[15,16,17,18,19,20,21]$.

\section{Fluid Dark Matter}

The Dark Matter might have a significant self-scattering cross-section $\sigma$, comparable to the neucleon-neucleon scattering cross section[15].Then in any halo, large or small, where the number of particles per unit area (the surface density) x $\sigma$ is greater than unity,colisionless amongst the Dark Matter particles leads to a complex evolution of the structure. During the initial phases of this process, which continues this lasts longer than the present age of the universe, the central densities decline in the desired fashion due to the scattering of Dark Matter particles. Also scattering strips the halos from small clump of Dark Matter orbiting larger structures, making them vulnerable to tidal stripping and reducing their number. 
We calculate the kinetic energy as constant for spiral shape galaxy when its particles interact with dark matter vortex.

1.1 By considering Lagrange's equation for particle where a, b, c are the coordinates of a particle at $\mathrm{t}=0$ and $\mathrm{x}, \mathrm{y}, \mathrm{z}$ are it's coordinates at $\mathrm{t}=\mathrm{t}$. If $\delta \mathrm{x} \delta \mathrm{y}$ $\delta z$ is the fluid element around this particle, the components of acceleration of this fluid elements are given by $\frac{\partial^{2} x}{\partial t^{2}}, \frac{\partial^{2} y}{\partial t^{2}}, \frac{\partial^{2} z}{\partial t^{2}}$. The velocity components of this particle at $\mathrm{t}=0$ are $\mathrm{u}, \mathrm{v}, \mathrm{w}$ and density of fluid is $\rho$ and pressure $\mathrm{p}$ and if we assume existence of a potential $\mathrm{V}$ corresponding to external forces, we have

$$
\frac{\partial^{2} x}{\partial t^{2}}=-\frac{\partial V}{\partial x}-\frac{1}{\rho} \frac{\partial p}{\partial x}
$$

similarly we obtain equations along $\mathrm{Y}$ and $\mathrm{Z}$ axis direction .The equation of continuity gives

$$
\rho \frac{\partial(x, y, z)}{\partial(a, b, c)}=\rho_{0}
$$

which constitute Lagrange's Hydrodynamical Equation .

\section{Cauchy's Integral}

Assuming that $\rho$ is a function of $p$, differentiating equation (2) and (3) with regard to $\mathrm{c}$ and $\mathrm{b}$ respectively and subtract and we obtain after writing $u, v, w$ for $\frac{\partial x}{\partial t}, \frac{\partial y}{\partial t}, \frac{\partial z}{\partial t}$ respectively

$$
\frac{\partial^{2} u}{\partial t \partial b} \frac{\partial x}{\partial c}-\frac{\partial^{2} u}{\partial t \partial c} \frac{\partial x}{\partial b}+\frac{\partial^{2} v}{\partial t \partial b} \frac{\partial y}{\partial c}-\frac{\partial^{2} v}{\partial t \partial c} \frac{\partial y}{\partial b}+\frac{\partial^{2} w}{\partial t \partial b} \frac{\partial z}{\partial c}-\frac{\partial^{2} w}{\partial t \partial c} \frac{\partial z}{\partial b}=0
$$

We now take a case when $\frac{\partial x}{\partial a}=1, \frac{\partial x}{\partial b}=\frac{\partial x}{\partial c}=0$. Now integrate with respect to $t$, we get by representing components of initial velocities as $u_{0}, v_{0}, w_{0}$

$$
\left(\frac{\partial w}{\partial y}-\frac{\partial v}{\partial z}\right) \frac{\partial(y, z)}{\partial(b, c)}+\left(\frac{\partial u}{\partial z}-\frac{\partial w}{\partial x}\right) \frac{\partial(z, x)}{\partial(b, c)}+\left(\frac{\partial v}{\partial x}-\frac{\partial u}{\partial y}\right) \frac{\partial(x, y)}{\partial(b, c)}=\frac{\partial w_{0}}{\partial b}-\frac{\partial v_{0}}{\partial c}
$$

writing

$$
\frac{\partial w}{\partial y}-\frac{\partial v}{\partial z}=2 \xi
$$

we obtain equations

$$
\begin{aligned}
& \frac{\partial u}{\partial z}-\frac{\partial w}{\partial x}=2 \eta \\
& \frac{\partial v}{\partial x}-\frac{\partial u}{\partial y}=2 \zeta
\end{aligned}
$$




$$
\begin{gathered}
\xi \frac{\partial(y, z)}{\partial(b, c)}+\eta \frac{\partial(z, x)}{\partial(b, c)}+\zeta \frac{\partial(x, y)}{\partial(b, c)}=\xi_{0} \\
\xi \frac{\partial(y, z)}{\partial(c, a)}+\eta \frac{\partial(z, x)}{\partial(c, a)}+\zeta \frac{\partial(x, y)}{\partial(c, a)}=\eta_{0} \\
\xi \frac{\partial(y, z)}{\partial(a, b)}+\eta \frac{\partial(z, x)}{\partial(a, b)}+\zeta \frac{\partial(x, y)}{\partial(a, b)}=\zeta_{0}
\end{gathered}
$$

where $\quad \xi, \eta, \zeta$ are the angular velocities.

Multiplying these equations by $\frac{\partial x}{\partial a}, \frac{\partial x}{\partial b}, \frac{\partial x}{\partial c}$ respectively and adding we get using the equation of continuity

$$
\frac{\xi}{\rho}=\frac{\xi_{0}}{\rho_{0}} \frac{\partial x}{\partial a}+\frac{\eta_{0}}{\rho_{0}} \frac{\partial x}{\partial b}+\frac{\zeta_{0}}{\rho_{0}} \frac{\partial x}{\partial c}
$$

similarly $\frac{\eta}{\rho}=\frac{\xi_{0}}{\rho_{0}} \frac{\partial y}{\partial a}+\frac{\eta_{0}}{\rho_{0}} \frac{\partial y}{\partial b}+\frac{\zeta_{0}}{\rho_{0}} \frac{\partial y}{\partial c}$

$$
\text { and } \frac{\zeta}{\rho}=\frac{\xi_{0}}{\rho_{0}} \frac{\partial z}{\partial a}+\frac{\eta_{0}}{\rho_{0}} \frac{\partial z}{\partial b}+\frac{\zeta_{0}}{\rho_{0}} \frac{\partial z}{\partial c}
$$

When velocity potential exists the motion is said to be irrotational and we therefore have from Cauchy's theorem that the motion of a fluid under conservative forces, if once irrotational, is always irrotational. When velocity does not exist, the motion is called rotational. Therefore in the case of irrotational motion, we have $\xi=\eta$ $=\zeta=0$. This is in accordance with theorem of permanence of irrotational motion at any instant, when the motion of fluid is irrotational; the circulation in all reducible circuits in the fluid vanishes. Circulation in any such circuit remains zero. Hence at any subsequent time, by Stokes theorem, we have

$$
\iint(1 \xi+m \eta+n \zeta) d s=0
$$

where $1, \mathrm{~m}, \mathrm{n}$ are direction cosines, the integration may be taken over any surface lying

wholly in the fluid and requires that

$\xi=\eta=\zeta=0$

at every point in the fluid and therefore the motion is always irrotational. 


\section{Vortex Motion}

So far we have confined our attention almost entirely to cases involving irrotational motion only. But most general displacement of a fluid involves rotation of which the components of angular velocity at a point $(\mathrm{x}, \mathrm{y}, \mathrm{z})$ are;

$$
\begin{aligned}
& \left(\frac{\partial w}{\partial y}-\frac{\partial v}{\partial z}\right) / 2=\xi \\
& \left(\frac{\partial u}{\partial z}-\frac{\partial w}{\partial x}\right) / 2=\eta \\
& \left(\frac{\partial v}{\partial x}-\frac{\partial u}{\partial y}\right) / 2=\zeta
\end{aligned}
$$

It is important to realize at the outset that some portion of fluid mass may possess rotation while other portions are moving irrotationally. Lines drawn are called vortex lines and portion of the fluid bounded by vortex lines called vortex filament or simply vortices and boundary of vortex filament is called vortex tube.

\section{Production of Vorticity}

If the dark matter is considered as viscous fluid, viscosity plays an important part in the production of circulation or vorticity, Vortex sheets are actually unstable. We have now seen that the production of such a sheet in perfect fluid is not inconsistent with the theory. When a stream of dark matter is obstructed by flat or bluff galaxy, the surface of discontinuity or vortex sheets behind the galaxy commonly roll up on themselves and produce a Karman Street of one or less concentrated vortices. When an aerofoil meets a stream and divides it, the two portions of the stream which pass above and below the galaxy meet again behind it; vortices produced by viscosity in a thin layer of the dark matter fluid surrounding the galaxy collect into a single vortex and move away from aerofoil leaving behind it a state of steady flow. The region of space which includes the body is cyclic and when the vortex in cast off behind the body, a circulation is setup round the body equal and opposite to that of the vortex so that the total circulation in a circuit which embraces the body and vortex remains zero.

\section{Kinetic Energy of a System of Vortices:}

The kinetic energy of dark matter fluid is T; where

$$
2 \mathrm{~T}=\rho \iiint\left(u^{2}+v^{2}+w^{2}\right) d x d y d z
$$

If the fluid is incompressible we may write equation of continuity

$$
\frac{\partial u}{\partial x}+\frac{\partial v}{\partial y}+\frac{\partial w}{\partial z}=-\frac{1}{\rho} \frac{D \rho}{D t}
$$

but if $V^{\prime}$ be the volume of a small element of fluid its mass $\rho V^{\prime}$ is variable, so that 
$\frac{D\left(\rho V^{\prime}\right)}{D t}=\frac{V^{\prime} D(\rho)}{D t}+\frac{\rho D\left(V^{\prime}\right)}{D t}=0$

hence;

$$
\frac{\partial u}{\partial x}+\frac{\partial v}{\partial y}+\frac{\partial w}{\partial z}=\frac{1}{V^{\prime}} \frac{D V^{\prime}}{D t}=\theta
$$

where $\theta$ denotes the expansion or rate of increase of volume at $(x, y, z)$. The expansion will cause extra terms in the expression for velocities; the expansion of an element $d x^{\prime} d y^{\prime} d z^{\prime}$ being equivalent to a simple source of strength $\frac{\theta}{4 \pi} d x^{\prime} d y^{\prime} d z^{\prime}$ at $\left(x^{\prime}, y^{\prime}, z^{\prime}\right)$. This gives rise to a velocity potential whose value at $(x, y, z)$ is

$$
\phi=\frac{1}{4 \pi} \iiint \frac{\theta^{\prime}}{r} d x^{\prime} d y^{\prime} d z^{\prime}
$$

and complete expression for the velocity are

$$
\begin{aligned}
& u=-\frac{\partial \phi}{\partial x}+\frac{\partial H}{\partial y}-\frac{\partial G}{\partial z} \\
& v=-\frac{\partial \phi}{\partial y}+\frac{\partial F}{\partial z}-\frac{\partial H}{\partial x} \\
& w=-\frac{\partial \phi}{\partial z}+\frac{\partial G}{\partial x}-\frac{\partial F}{\partial y}
\end{aligned}
$$

by which

$$
2 T=\rho \iiint\left\{u\left(-\frac{\partial \phi}{\partial x}+\frac{\partial H}{\partial y}-\frac{\partial G}{\partial z}\right)+v\left(-\frac{\partial \phi}{\partial y}+\frac{\partial F}{\partial z}-\frac{\partial H}{\partial x}\right)+w\left(-\frac{\partial \phi}{\partial z}+\frac{\partial G}{\partial x}-\frac{\partial F}{\partial y}\right)\right\} d x d y d z
$$

Where the surface integral extends to the whole boundary of the fluid and the triple integral are taken throughout the volume.

If we suppose that the fluid dark matter extends to infinity and is at rest there and that the vortices are all within a finite distance of the origin, then the first integral vanishes, the second is zero because $\nabla^{2} \phi=0$, and the third is zero because at point on the infinitely distant boundary F, G, $\mathrm{H}$ are ultimately of the order $\frac{1}{R^{2}}$, and $\mathrm{u}, \mathrm{v}, \mathrm{w}$ of order $\frac{1}{R^{3}} ;$ therefore

$$
T=\rho \iiint(F \xi+G \eta+H \zeta) d x d y d z
$$


We find values of $\mathrm{F}, \mathrm{G}$ and $\mathrm{H}$, in terms of $\xi$, $\eta$ and $\zeta$. The fluid being incompressible, the flow across any two surfaces having the same curve for boundary will be the same, and therefore depends only on the form of the boundary. If we assume that this flow can be represented by line integral round the boundary, we get

$$
\iint(l u+m v+n w) d s=\int(F d x+G d y+H d z)
$$

where $\mathrm{F}, \mathrm{G}, \mathrm{H}$ are component of a certain vector.

But

$$
\int(F d x+G d y+H d z)=\iint\left\{l\left(\frac{\partial H}{\partial y}-\frac{\partial G}{\partial z}\right)+m\left(\frac{\partial F}{\partial z}-\frac{\partial H}{\partial x}\right)+n\left(\frac{\partial G}{\partial x}-\frac{\partial F}{\partial y}\right)\right\} d S
$$

Hence we must have , $u=\frac{\partial H}{\partial y}-\frac{\partial G}{\partial z}, v=\frac{\partial F}{\partial z}-\frac{\partial H}{\partial x}, w=\frac{\partial G}{\partial x}-\frac{\partial F}{\partial y}$

or as it may be expressed more briefly

$u, v, w=\operatorname{curl}(\mathrm{F}, \mathrm{G}, \mathrm{H})$

It is clear that the values of $u, v, w$ given by equation 1 satisfy the equation of continuity; and

substituting in the values for $\xi, \zeta, \eta$ we get

$$
\left(2 \xi=\frac{\partial w}{\partial y}-\frac{\partial v}{\partial z}=\frac{\partial}{\partial x}\left(\frac{\partial F}{\partial x}+\frac{\partial G}{\partial y}+\frac{\partial H}{\partial z}\right)-\nabla^{2} F\right)
$$

and similar expression for $2 \eta$ and $2 \zeta$.

Hence the assumptions of equation 1 will be justified if we can find F, G, H so as to satisfy

the equations 3 and 4

$$
\begin{aligned}
& \frac{\partial F}{\partial x}+\frac{\partial G}{\partial y}+\frac{\partial H}{\partial z}=0 \\
& \nabla^{2} \mathrm{~F}=-2 \xi, \nabla^{2} \mathrm{G}=-2 \eta, \nabla^{2} \mathrm{H}=-2 \zeta
\end{aligned}
$$

The above equations can be satisfied by assuming $\mathrm{F}, \mathrm{G}$ and $\mathrm{H}$ to be potential functions due to distribution of gravitating matters of volume densities $\frac{\xi}{2 \pi}, \frac{\eta}{2 \pi}, \frac{\zeta}{2 \pi}$ respectively. We then have 


$$
\begin{aligned}
& F=\frac{1}{2 \pi} \iiint \frac{\xi^{\prime}}{r} d x^{\prime} d y^{\prime} d z^{\prime} \\
& G=\frac{1}{2 \pi} \iiint \frac{\eta^{\prime}}{r} d x^{\prime} d y^{\prime} d z^{\prime} \\
& H=\frac{1}{2 \pi} \iiint \frac{\zeta^{\prime}}{r} d x^{\prime} d y^{\prime} d z^{\prime}
\end{aligned}
$$

Where

$$
r^{2}=\left(x-x^{\prime}\right)^{2}+\left(y-y^{\prime}\right)^{2}+\left(z-z^{\prime}\right)^{2}
$$

And

$\xi^{\prime}, \zeta^{\prime}, \eta^{\prime}$

are components of spin of the element $\mathrm{dx}^{\prime} \mathrm{dy}^{\prime} \mathrm{dz}^{\prime}$ at $\left(\mathrm{x}^{\prime}, \mathrm{y}^{\prime}, \mathrm{z}^{\prime}\right)$. Here range is taken as extending for whole fluid, though the integrand is zero at all points at which there are no spin.

Thus by putting the values of $\mathrm{F}, \mathrm{G}, \mathrm{H}$ we get

$$
T=\frac{\rho}{2 \pi} \iiint \iiint \frac{\left(\xi \xi^{\prime}+\eta \eta^{\prime}+\zeta \zeta^{\prime}\right)}{r} d x d y d z d x^{\prime} d y^{\prime} d z^{\prime}
$$

where each volume integral extends through the whole space occupied by the vortices.

\section{Kinetic Energy Constant:}

We can also show that kinetic energy of dark matter fluid is constant when no extraneous forces act. The equation of motion is

$$
\frac{D}{D t}(u, v, w)=-\frac{1}{\rho}\left(\frac{\partial p}{\partial x}, \frac{\partial p}{\partial y} \frac{\partial p}{\partial z}\right)
$$

Multiplying these by $u, v, w$ and adding we get

$$
\frac{1}{2} \rho \frac{D}{D t}\left(u^{2}+v^{2}+w^{2}\right)=-\left(u \frac{\partial p}{\partial x}+v \frac{\partial p}{\partial y}+w \frac{\partial p}{\partial z}\right)
$$

Multiplying by $d x d y d z$ and integrate over any region we get

$$
\begin{aligned}
& \frac{D T}{D t}=-\iiint\left(u \frac{\partial p}{\partial x}+v \frac{\partial p}{\partial y}+w \frac{\partial p}{\partial z}\right) d x d y d z \\
& =\iint(l u+m v+n w) p d S
\end{aligned}
$$

Integrated over the boundary of the region.

Therefore, at great distance $\mathrm{R}$ from the vortices $\mathrm{p}$ will be finite and $l u+m v+n w$ of order $\frac{1}{R^{3}}$ while dS in of order $\mathrm{R}^{2}$. Hence the expression for $\frac{D T}{D t}$ vanishes and we have

$\mathrm{T}=$ constant 
In fig: 1 a graph of distance from center of galaxy to the kinetic energy describe that kinetic energy becomes constant at larger distances[22]. Kinetic energy is calculated with respect to local comoving frame [23].

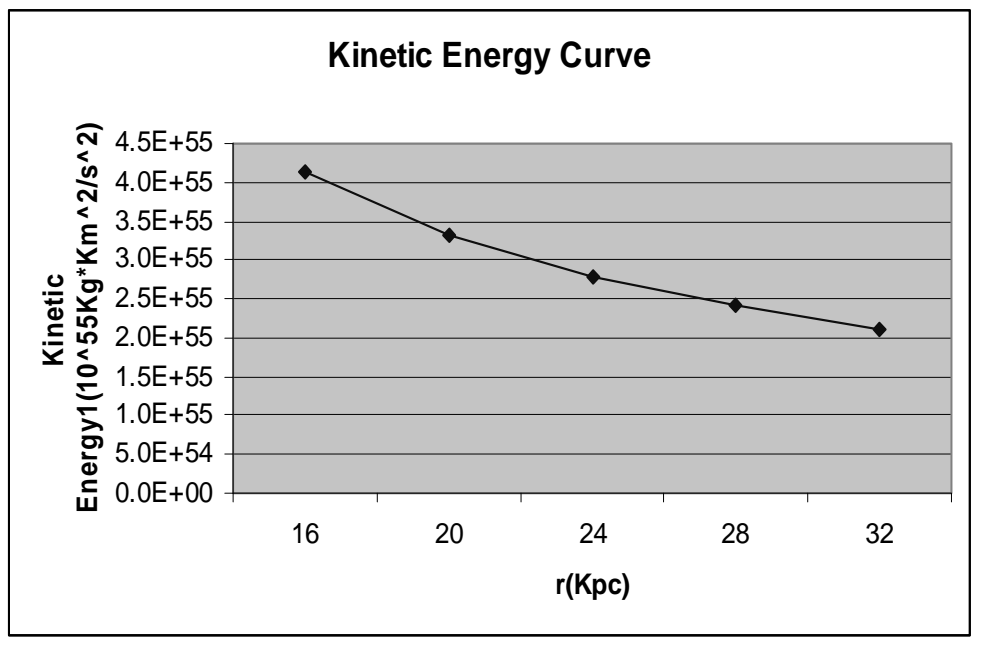

Figure 1: Kinetic Energy of Milky way Galaxy is shown which becomes constant at larger distances due to presence of dark matter.

Now we turn to see how vortices are form in Axion condensate [24].

\section{Vortex in axion condensate as a dark matter halo:}

The two main candidates for cold dark matter are namely the axion and neutralino $[25,26]$. If axions have low mass, thus preventing other decay modes, axion theories predict that the universe would be filled with cold Bose-Einstein condensate of primordial axions. If axions have low mass, as a result preventing other decay modes, axion theories envisage that the universe would be filled with cold Bose- Einstein condensate of nonrelativistic primordial axions.Many experiments were suggested for direct detection of Axions such as: Axion Dark Matter Experiment (ADMX) [27], CAST at CERN, The Large Scale US Axion Search at Lawrence Livermore National Laboratory and PVLAS Experiment at INFN etc. ADMX searches for weakly interacting axions in the dark matter halo of our galaxy. They investigate the possibility of explanation of flat velocity curves of spiral galaxies in terms of axion condensate which can be presented in the Universe since the Peccei-Quinn phase transition. In their discussion they use the Gross-Pitaevski equation in an expanding FRW universe.

$$
i \hbar\left(\frac{\partial}{\partial t}+\frac{3}{2} \frac{\dot{a}(t)}{a(t)}\right) \phi(\vec{r}, t)=\left(-\frac{\hbar^{2}}{2 m} \frac{1}{a^{2}(t)} \nabla^{2}+U(\vec{r})+g^{2}|\phi(\vec{r}, t)|^{2}\right) \phi(\vec{r}, t),
$$


where $U(\vec{r})$ is the external potential, $g^{2}$ is the coupling constant between axions and $a(t)$ is the scale factor. Here we describe the condensate by one particle wave function $\phi(\vec{r}, t)$

The circulation in condensate is expressed by [10]

$$
\Gamma=\oint_{C} \vec{v} \cdot d \vec{r}=\frac{\hbar}{m} 2 \pi l,
$$

where $\ell$ is an integer called topological charge. $C$ denotes any contour around a vortex. When there is no vortex, $\ell=0$ and the circulation vanishes. When the condensate is inside a rotating environment the vortex is formed. In the interacting condensate vortices with $\ell>1$ are unstable and decay to the vortices with $\ell=1$. So in a realistic situation we have a net of elementary vortices $(\ell=1)$ which are stable.

They solve equation to obtain the density distribution in the vortex. Considering Bessel function solution for $\ell=0, \ell=1$ and for a spherical distribution the coordinate $\mathrm{r}$ in not a physical distance, which is $\mathrm{R}=\mathrm{a} \cdot \mathrm{r}$. they calculate the velocity rotation curve from the relation

$$
v(R)=\sqrt{\frac{G M(R)}{R}}
$$

where $\mathrm{M}(\mathrm{R})$ is the mass function and is expressed as

$$
M(R)=4 \pi \int_{0}^{R} R^{\prime 2} \rho\left(R^{\prime}\right) d R^{\prime}
$$

Figure (2) shows the velocity curve for axion condensate with assumed $\sqrt{ } \lambda / \mathrm{a}=0.2$ kpc.

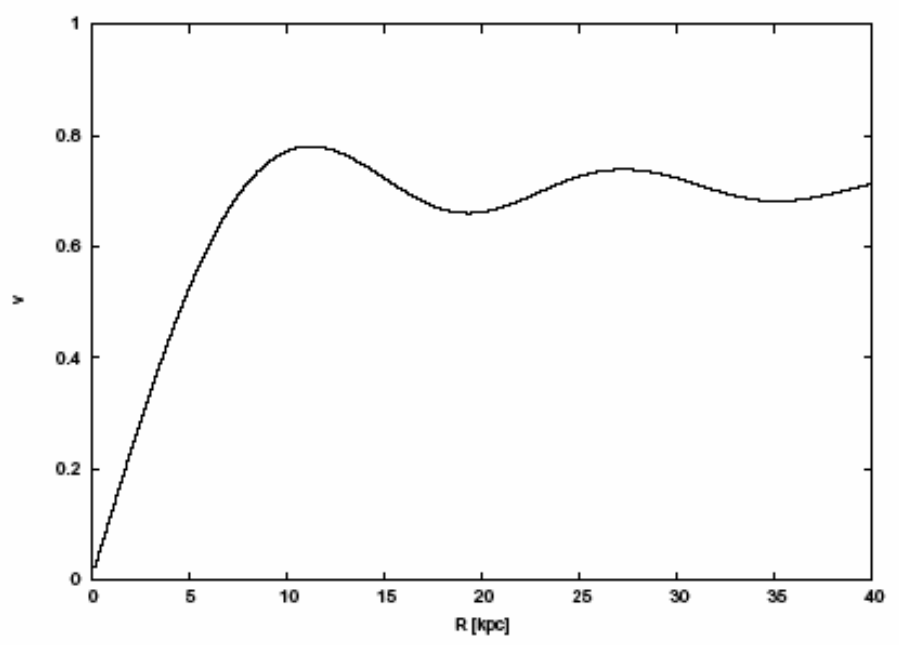

Figure 2: The contribution to the galactic velocity curve given by the axion condensate.

This result describes the observed galaxy velocity curves quite well; we can see the characteristic change to a plateau in the rotation curve. For further analysis they 
choose the point of transition to plateau in the rotation curve as it observed, which is estimated to take place for Rtrans $\simeq 10 \mathrm{kpc}$ ( $\mathrm{R}$ is the physical value, measured today). Very similar results can be obtained considering the vortex solution with $\ell=1$. The velocity curves also exhibit a plateau, like the solution with $\ell=0$. The only difference is that we now have the angle dependence $\rho \sim \cos 2 \theta$. This produces, like for each vertex solution, distortions from the spherical shape of a dark matter halo. The dispersion of velocities of axions is very small [28] but it can produce normalised states. Assuming this distribution we obtain a normalised wave function as

$$
\Psi(\vec{r}, t)=\frac{k_{0}^{3 / 2}}{2 \sqrt{\pi a^{3} \sqrt{\frac{\pi}{2}}}} \frac{e^{-\left(r / r_{0}\right)^{2}}}{\left.1+\left(\frac{\hbar k_{0}^{2}}{8 m H a^{2}}\right)^{2}\right]^{3 / 4}},
$$

where

$$
r_{0}=\frac{1}{k_{0}} \sqrt{1+\left(\frac{\hbar k_{0}^{2}}{8 m H a^{2}}\right)^{2}}
$$

The velocity curve for this model with $\operatorname{ar} 0=10 \mathrm{kpc}$ is shown in Figure 3. As we can see in this figure, after taking a normalisable superposition of states, the velocity curve no longer has a plateau. For higher distances the velocity starts to decrease.

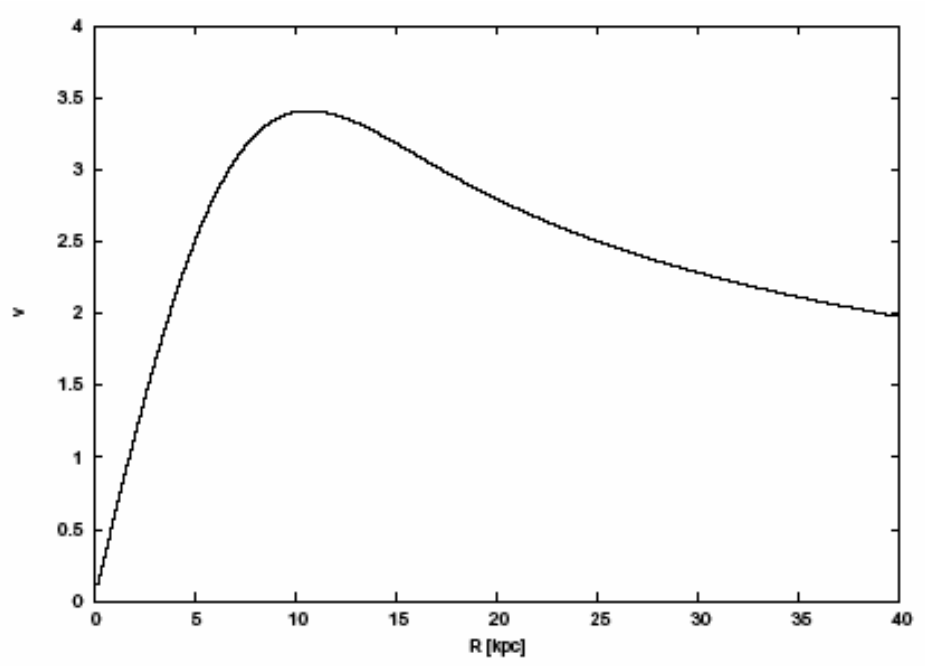

Figure 2: The contribution to the galactic velocity curve given by the axion condensate with $\operatorname{ar}_{0}=10 \mathrm{kpc}$.

\section{Conclusion}

Our result in figure (1) shows that velocity of spiral shape galaxy first decrease and then become constant at larger distances from galactic center. Our result also shows that velocity dispersion curve is a flat curve. The model used by [24] also shows the same types of conclusion what we investigated in our work. 


\section{Acknowledgment}

We express our gratitude to Chairman of Prasar Shikshan Evam Seva Sansthan Mr. Praveen Vashishtha under whose continuous support and encouragement this work was done. We also wish to thank Director Dr. (Prof.) Vivek Bansod of M.I.T.S. to provide us facilities and guidance in various ways. We are also thankful to $\boldsymbol{D r}$. (Prof.) S.B. Shrivastava Head School of Studies in Physics, Vikram University, Ujjain.

\section{References}

[1] F.Zwickey,ApJ 86,217(1937)

[2] M.S.Roberts,A.H.Rots,Astr.and Astrophs.26,483(1973)

[3] J.P.Ostriker,P.J.E.Peebles,A.Yahil,ApJ letters 193,L1(1974)

[4] J.Enasto,A.Kaasik,E.Saar,Nature 250,309(1974)

[5] V.C.Rubin,N.Thonnard,W.K.Ford Jr.,ApJ letters 225,L107(1978)

[6] N.Dalal,C.S.Kochanek,ApJ572,25(2002)

[7] G.Toth,J.P.Ostriker,ApJ 389,5(1992)

[8] A.S.Font,J.F.Navarro,J.Stadel,T.Quinn,ApJ 563,L1(2001)

[9] J.A.Tyson,G.P.Kochansky,I.P.Dell' Antonio,ApJ letters 498,L107(1998)

[10] J.J.Binney,N.W.Evans,MNRAS,327,L27(2001)

[11] R.Dave',D.N.Spergel,P.Steinhardt,B.Wandelt,ApJ 547,574(2001)

[12] F.C.Van den Bosch,B.E.Bobertson,J.J.Dalcanton,W.J.G.de Bok,ApJ 119,1579 (2000)

[13] F.Stoehr, S.D.M.White,G.Tormen,V.Springel MNRAS 335,L84(2002)

[14] J.T.Kleyna,M,Wilkinson,G.Gilmore,W.N.Evans,astro-ph/0304093 (2003)

[15] D.N.Spergel, P.Steinhardt,Phys.Rev.Lett.84,3760(2000)

[16] W.H.Besant, A.S.Ramsey, A Treatise On Hydromechanics I-II ELBSG.Bell\&Sons246 (1965)

[17] Niayesh Afshordi, arXiv: astro-ph/0610336(2006)

[18] J.P. Ostriker, \& P.J. Steinhardt, Science, 300, pp 1909-1914 (2003). 264.

[19] Stevan R. Furlanetto,A.Loeb,astro-ph 0107567 (2001)

[20] Ben Moore et. al., arXiv: astro-ph 002308 (2000)

[21] P. J. E. Peebles , arXiv: astro-ph/0002495

[22] R.Sharma, G.K.Upadhyaya.abs.proc.of conf. DARK-2007 VI International Heidelberg Conference on Dark Matter in Astro Particle Physics (2007).

[23] Mark H.Jones, Robert J Lambourne, David J. Adams,"An Introduction to Galaxies and Cosmology"Cambridge Univ.Press 298 (2004)

[24] J.Mielczarek, T. Stachowiak, M.Szydlowski, arXiv: astro-ph /0705.3017v2

[25] G. Lazarides, arXiv:hep-ph/0601016

[26] P. Gondolo, NATO Sci. Ser. II 187, 279 (2005), arXiv:astro-ph/0403064

[27] L. D. Duffy et al., Phys. Rev. D 74, 012006 (2006), arXiv:astro-ph/0603108

[28] P. Sikivie, arXiv:astro-ph/0610440 